\title{
BMJ Open Cohort profile: the St Vincent's Melbourne Arthroplasty Outcomes (SMART) Registry, a pragmatic prospective database defining outcomes in total hip and knee replacement patients
}

\author{
Daniel Gould (D) , ${ }^{1}$ Sharmala Thuraisingam (D) ,,2 Cade Shadbolt (D) , \\ Josh Knight (D) , Jesse Young (D) , , ${ }^{4,5}$ Chris Schilling (D) , , Peter F Choong, ${ }^{1,6}$ \\ Michelle M Dowsey (D) ${ }^{1,6}$
}

To cite: Gould D,

Thuraisingam S, Shadbolt C, et al. Cohort profile: the St Vincent's Melbourne Arthroplasty Outcomes (SMART) Registry, a pragmatic prospective database defining outcomes in total hip and knee replacement patients. BMJ Open 2021;11:e040408. doi:10.1136/ bmjopen-2020-040408

- Prepublication history and additional materials for this paper is available online. To view these files, please visit the journal online (http://dx.doi. org/10.1136/bmjopen-2020040408).

Received 14 May 2020

Revised 01 December 2020 Accepted 07 December 2020

Check for updates

(c) Author(s) (or their employer(s)) 2021. Re-use permitted under CC BY-NC. No commercial re-use. See rights and permissions. Published by BMJ.

For numbered affiliations see end of article.

Correspondence to Dr Michelle M Dowsey; mmdowsey@unimelb.edu.au

\section{ABSTRACT}

Purpose The St Vincent's Melbourne Arthroplasty Outcomes (SMART) Registry is an institutional clinical registry housed at a tertiary referral hospital in Australia. The SMART Registry is a pragmatic prospective database, which was established to capture a broad range of longitudinal clinical and patient-reported outcome data to facilitate collaborative research that will improve policy and practice relevant to arthroplasty surgery for people with advanced arthritis of the hip or knee. The purpose of this cohort profile paper is to describe the rationale for the SMART Registry's creation, its methods, baseline data and future plans for the Registry. A full compilation of the data is provided as a reference point for future collaborators.

Participants The SMART Registry cohort comprises over 13000 consecutive arthroplasty procedures in more than 10000 patients who underwent their procedure at St Vincent's Hospital Melbourne, since January 1998. Participant recruitment, data collection and follow-up is ongoing and currently includes up to 20 years follow-up data.

Findings to date SMART Registry data are used for clinical audit and feedback, as well as for a broad range of research including epidemiological studies, predictive statistical modelling and health economic evaluations. At the time of writing, there were 46 publications from SMART Registry data, with contributions from more than 67 coauthors.

Future plans With the recent linking of the SMART Registry with Medicare Benefits Schedule and Pharmaceutical Benefits Scheme data through the Australian Institute of Health and Welfare, research into prescribing patterns and health system utilisation is currently underway. The SMART Registry is also being updated with the Clavien-Dindo classification of surgical complications.
Strengths and limitations of this study

All elective hip and knee arthroplasty patients at the hospital are included.

- A range ofpatient-reported outcome measures are recorded with long-term follow-up and high proportion of patients participating in follow-up, with $>98 \%$ capture at 12 months.

- While the Registry is institutional, outcomes have compared against several international settings and our data were comparable. However, this is no guarantee that findings from analysis of St Vincent's Melbourne Arthroplasty Outcomes Registry data will be applicable to a particular setting of interest.

- The Registry is not linked to state-based databases such as the Victorian Emergency Minimum Dataset and the Victorian Admitted Episodes Dataset, therefore, it is not possible to determine with absolute certainty whether registry patients were subsequently admitted to other hospitals, or experienced an emergency department visit at another hospital.

- The institution does not currently have a linkable electronic medical record, and therefore, manual extraction of data is required.

\section{INTRODUCTION}

Primary aims which motivated the creation of the registry

Total hip and knee joint arthroplasty (TJA) are both highly effective procedures for reducing pain, improving function, and enhancing quality of life in patients with advanced arthritis. ${ }^{12}$ Although it is associated with a significant cost to healthcare systems, the rate of TJA continues to grow in Australia and globally. ${ }^{3-7}$ The St Vincent's Melbourne Arthroplasty Outcomes (SMART) Registry (from here on referred to as "the 


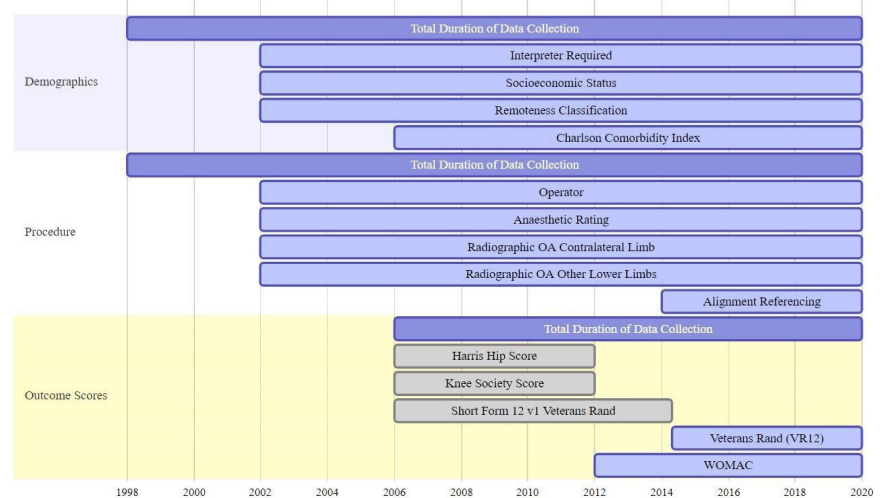

Figure 1 Changes to the capture of SMART Registry measures over time. Total duration of data collection', the year in which recording of the given category of variables commenced-highlights when other variables were added to this category; Anaesthetic Rating, American Society of Anaesthesiologists Classification; OA, osteoarthritis; SMART, St Vincent's Melbourne Arthroplasty Outcomes; WOMAC, Western Ontario and McMaster Universities Osteoarthritis Index.

Registry') was created in 2005 to identify patient risk factors for poor response to surgery and to identify gaps in the uptake of evidence-based practice. ${ }^{8-11}$ It captures relevant data on elective arthroplasty surgery of the hip and knee, including utilisation rates, outcomes, changes in patient characteristics over time, complication rates and economic cost. The Registry aims to: (1) provide a better understanding of the demographic and clinical characteristics of arthroplasty recipients, (2) detail all aspects of the surgical procedure, (3) monitor the safety and efficacy of arthroplasty surgery, (4) provide data on the performance of individual surgeons, (5) drive quality improvement within St Vincent's and generate evidence which is generalisable to the national and international patient population, and (6) allow for comparisons between this institutional registry and other arthroplasty registries. Patient-reported outcome measures (PROMs) data have been collected prospectively in the Registry since 1 January 2006 to gain a more complete understanding of outcomes (see figure 1 and online supplemental file 1$).{ }^{12}$ Since there is a relatively low incidence of major complications in arthroplasty surgery, such as revisions and mortality, the registry is also ideally situated to provide rigorous long-term monitoring that is not feasible with randomised controlled trials in medical implant recipients. ${ }^{13}$ The purpose of this cohort profile paper is to comprehensively and transparently report the data collection processes, as well as evolution of our institutional registry. In doing so, the research community can assess the rigour, reliability and generalisability of research arising from the registry and if relevant, use this information as a stimulus for wider collaborations

\section{Current state of the registry}

As of 30 June 2018, the registry comprises data from 13155 arthroplasty procedures in 10294 patients. Data collection is continuous and ongoing, comprising a range of clinical, demographic, prosthesis-specific and operation-specific, and patient-reported variables.

\section{COHORT DESCRIPTION}

\section{Setting and location}

The registry captures data for all patients who undergo elective hip and knee arthroplasty at St Vincent's Hospital, a tertiary referral centre for TJA surgery in Melbourne, Australia, which receives referrals from all of Victoria. In 2018, the population of Victoria was estimated at 6.3 million people, with $77 \%$ residing in Melbourne. ${ }^{14}$

\section{Relevant dates}

On 1 January 2005, as part of a $\mathrm{PhD}$ project, the registry was established. Data collection has been prospective since 1 January 2005, with retrospective data collection for cases from 1998 to 2004. The clinical data of all patients who underwent elective arthroplasty dating back to 1 January 1998 were extracted and added to the registry. This was the earliest available date that data could be reliably extracted from patient medical records. Cases were prospectively added from 1 January 2005 and routine prospective collection of PROMs was introduced from 1 January 2006. Any ad hoc PROM collection related to specific studies that occurred prior to 1 January 2006 were also added to the Registry. Data collection is ongoing and approximately 750 to 800 new cases have been added to the registry in each of the five calendar years 2015-2019. This represents $3 \%$ of cases performed in the state of Victoria. $^{15}$

\section{Eligibility criteria and how patients are recruited}

All patients who undergo elective total hip or knee arthroplasty at St. Vincent's Hospital (Melbourne) are eligible for inclusion on the registry. SMART captures data on primary elective total hip and knee arthroplasty, as well as unicompartmental and patellofemoral knee, and revision hip and knee arthroplasty procedures. Informed consent is currently obtained from participants preadmission (ie, prior to their admission assessment) using an 'opt-out' approach, which has been ratified by the St Vincent's Hospital Melbourne (SVHM) ethics committee. The form is attached as online supplemental file 2. Specifically, when patients are scheduled for a preadmission assessment in preparation for arthroplasty surgery, they are sent an information pack containing the opt-out consent form, which is available in 11 languages: English, Arabic, Croation, Greek, Italian, Macedonian, Simplified Chinese, Traditional Chinese, Spanish, Turkish and Vietnamese. The Registry has $100 \%$ capture of elective arthroplasty procedures and $<0.1 \%$ of patients opt-out of completing PROMs.

\section{Description of data collection methods and follow-up}

Data collected includes patient demographics, current diagnoses and history of comorbidities such as cancer 


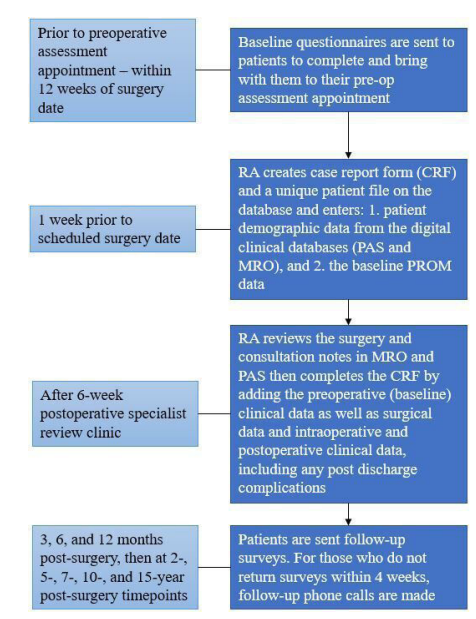

Figure 2 Flow chart-baseline data collection and follow-up. MRO, Medical Records On-line; PAS, Patient Administration System; PROM, patient-reported outcome measure; RA, research assistant.

resection, and confounders such as smoking history (see online supplemental file 1) for the specific variables collected and the source of each variable). Figure 2 outlines the process of baseline and follow-up data collection. Information is initially obtained by resident doctors in preadmission clinic and via correspondence with other health professionals involved in the patient's care. For example, comorbidities recorded during the preadmission clinic assessment are cross referenced against anaesthetist assessment recorded on the day of surgery. Preoperative PROM data are collected by a research assistant (RA) prior to surgery. Baseline patient clinical data are extracted from the St Vincent's digital clinical databases-Medical Records On-line and Patient Administration System-by an RA after the 6-week postoperative follow-up appointment, which they subsequently add to the patient's Registry file. Since the inception of the Registry, the baseline variables include: age; sex; body mass index (BMI) - measured on the day of surgery by the perioperative nursing team and classified according to WHO definitions as underweight (BMI $<18.5 \mathrm{~kg} / \mathrm{m}^{2}$ ), normal weight (BMI $18.5-24.9 \mathrm{~kg}$ / $\mathrm{m}^{2}$ ), overweight $\left(25.0-29.9 \mathrm{~kg} / \mathrm{m}^{2}\right)$, obese class I (BMI $30-34.9 \mathrm{~kg} / \mathrm{m}^{2}$ ), obese class II (BMI $35-39.9 \mathrm{~kg} / \mathrm{m}^{2}$ ) or obese class III $\left(\right.$ BMI $\left.\geq 40 \mathrm{~kg} / \mathrm{m}^{2}\right)$; a measure of comorbidity status obtained from the preoperative anaesthetic notes taken on the day of surgery according to the American Society of Anesthesiology (ASA) Classification; patient postcode is used to determine Socioeconomic Index for Area and Accessibility/Remoteness Index Australia classification-the postcode is mapped to these classifications by a trained RA, who enters them into the case report form (CRF) (see online supplemental file 3a,b) and the Registry database; aetiology; radiographic OA severity, graded using the KellgrenLawrence classification system applied to the preoperative plain films, taken within 6 months of surgery. ${ }^{16-23}$ These are collected in line with the goals of the registry as per the rationale described by Gliklich et al. ${ }^{24}$ Hospital cost data, including surgery, imaging, pharmacy and theatre costs, are currently available for years 2006-2007 and 2012-2016 from the Hospital's Decision Support Unit. All complications that represent a clinically meaningful alteration to the patient's postoperative course are recorded, in line with guidelines issued by the Royal Australasian College of Surgeons. ${ }^{25}$ A clinically meaningful alteration includes, but is not limited to, investigations, interventions, delayed discharge, representation and/or readmission to the hospital, and revision surgery. This broad capture of complications enables classification systems, such as the Clavien-Dindo system, to be applied to the SMART Registry because detailed data are rigorously routinely collected.

We achieve approximately $98 \%$ follow-up of PROMs at 1 year, $95 \%$ at 2 years, $85 \%$ at 5 years and $75 \%$ at 7 years. Lost to follow-up is almost exclusively due to patient death, with $<1 \%$ at any timepoint due to other reasons.

A range of surgery-related and prosthesis-related variables are recorded including surgical approach, alignment referencing technique, type of anaesthesia, wound drain output and surgeon level of training. Prosthesisrelated variables include implant brand, type of cement (if used), size of components, number of bone screws used (if applicable), femoral offset (hips) and degree of constraint (knees). Full details of the variables captured are available in the data dictionary (online supplemental file 1).

Revision arthroplasty is defined in line with the Australian Orthopaedic Association National Joint Replacement Registry (AOANJRR). ${ }^{26}$ Any further intervention required for the prosthesis, such as liner exchange, stem replacement are included as a revision procedure, which is recorded both as a complication related to the primary procedure and as a procedure of its own such that details specific to the revision surgery are captured comprehensively with the same rigour as for primary procedures. Washout and debridement for infection are recorded elsewhere as complication a complication rather than revision. The following measures are undertaken to maximise the capture of revision surgeries:

1. Patients are contacted for PROM surveys, at which time they are asked about any additional procedures related to their arthroplasty.

2. The operating theatre list is scrutinised each week in order to identify patients booked for revision surgery.

3. Data on revision procedures that are performed elsewhere are captured because the treating hospital makes a request to our institution for details of the components used during the index procedure as part of their planning for the revision procedure.

4. While individual patient data are not available from the AOANJRR, aggregate data are available which facilitates comparison between SMART Registry revision rates and national revision rates in order to flag whether there is the potential for substantial under-reporting of revision procedures. 
Over time, the nature of the research questions able to be answered using the data has developed as a result of changes and improvements in Registry data capture. Figure 1 presents measures in the registry for which the capture has changed over time in order to broaden the scope of research that can be conducted using the data. Routine collection of baseline quality of life and condition-specific questionnaires for knee $(\mathrm{n}=4722)$ and hip $(\mathrm{n}=4393)$ arthroplasty, commenced from 1 January 2006 onward. Since then, one condition-specific and one quality of life measure has been routinely collected presurgery and at the follow-up time points (see online supplemental file 1). The Knee Society Score (KSS) contains both patient-reported and surgeon-reported components. ${ }^{27}$ The scoring system is both clinician derived and patient derived and consists of a knee subscale completed by the surgeon, and satisfaction, functional activities and expectation subscales which are completed by the patient, with higher scores indicating better outcomes. ${ }^{28}$ The Harris Hip Score (HHS) was developed to assess the results of hip surgery and contains 10 items covering the domains of pain (one item, 0-44 points), function (seven items, 0-47 points), absence of deformity (one item, 4 points) and range of motion (two items, 5 points). ${ }^{29}$ The maximum score is 100 points, with higher scores indicating better results. Commencing on 1 January 2012, the HHS and KSS condition-specific questionnaires were superseded by the Western Ontario and McMaster Universities Osteoarthritis Index (WOMAC) for two main reasons: (1) to solely capture pain and function from the patient perspective as opposed to using questionnaires that give weight to the clinician perspective and (2) the use of the WOMAC has been endorsed by peak international bodies (Osteoarthritis Research Society International, Outcome Measures in Rheumatology as a valid measure for use in $\mathrm{OA}$ and TJA in multiple populations and is available in $>80$ languages. ${ }^{30}$ The WOMAC is a valid 24-item measure of pain and function for people with knee and hip osteoarthritis (OA) and for TJA. ${ }^{31-36}$ The WOMAC consists of three subscales that measure pain $(0-20)$, joint stiffness $(0-8)$ and physical function (0-68), with a lower score representing a better outcome. The Veterans RAND 12-item Health Survey (VR-12), a health-related quality of life instrument which measures well-being with a Physical Component Score (PCS) and Mental Component Score (MCS), is also administered. ${ }^{34} 3738$ The VR-12 PCS comprises four scales: physical functioning, role-physical, bodily pain and general health; the MCS also comprises four scales: vitality, social functioning, role emotional and mental health. Higher scores on the VR-12 indicate better quality of life. An overall utility score can also be calculated. $^{39}$

Introduced on 1 January 2006, the Charlson Comorbidity Index (CCI) is calculated from the data obtained from the preoperative anaesthetic notes taken on the day of surgery, and provides an accurate prediction of mortality based on weightings of certain comorbidities. ${ }^{21-23} 4041$ Anaesthetists independently assess patients through history and examination as well as medical record review of presurgery assessments performed by the resident medical officer in preparation for surgery, for presence of active comorbidities, and previous cancer diagnoses.

\section{Data linkage}

Baseline and follow-up data have been probabilistically linked with MBS federally subsidised healthcare and PBS medication dispensing records. The data linkage process was carried out by the Australian Institute of Health and Welfare Data Linkage Unit and involved a probabilistic linkage procedure consisting of 19 passes with clerical review for 'possible' record links. Each pass consisted of deterministic pairwise matching of records from SMART and Medicare Enrolment File (MEF) on selected blocking variables. The MEF has been described elsewhere, and the version used for this linkage included all Medicare customers registered from the foundation of Medicare (1 February 1984) to 30 December $2018 .{ }^{42}$ Weights were calculated based on probabilities of agreement and disagreement for the blocking and match variables for each respective match pair in the block. The blocking and match variables were based on surname, given name(s), postcode and day, month and year of birth. An optimal threshold algorithm was used to determine the optimal record pair.

The first of five annual linkages occurred over September 2019, and four additional annual linkages have already been approved. Annual linkages are funded through the National Health \& Medical Research Council Centre (NHMRC) of Research Excellence in Total Joint Replacement. The planned date of these future linkages is due in October each year. Access to the linked data has been approved from 8 May 2018 to 31 December 2028 , with the opportunity for additional annual linkages within this period depending on available funding.

The MBS records contain information on services that qualify for a benefit under the Health Insurance Act 1973, for which a claim has been processed. Briefly, MBS records include information about type of service provided, benefits paid and patient characteristics (such sex, year of birth and geographical region). ${ }^{43} 44$

The PBS data collection contains information on prescription medicines that qualify for a benefit under the National Health Act 1953, and for which a claim has been processed. The database comprises information about the PBS scripts and payments, patients (such as a patient identifier, beneficiary status and patient location), prescribers and dispensing pharmacies. ${ }^{45-47}$

\section{Data management and quality assurance}

Data entry and questionnaire follow-up is overseen by a dedicated Registry Coordinator, aided by two RAs. Data are housed on a dedicated orthopaedic research software system, Standardised Orthopaedic Clinical Research and Treatment Evaluation Software (Socrates). ${ }^{48}$ Registry data are audited annually by the principal investigator. 
Mortality data are checked against data from the Registrars of Births, Deaths and Marriages via the AOANJRR. ${ }^{26}$ The SMART Registry is separate to the AOANJRR, but patients undergoing arthroplasty at St Vincent's are also included in the National Registry. One of the major differences between the National Registry and SMART Registry is the duration of PROMs data collection, which commenced in 2018 for the National Registry with a preoperative and 6-month postoperative PROM survey, while the SMART Registry has been collecting longitudinal PROMs data comprehensively since 2006 (see online supplemental file 1) ${ }^{49}$ Individual surgeons at SVHM are also able to use peer benchmarking of their surgical, clinical and patient-reported outcomes, by comparing their own data with aggregate, deidentified data from the other SVHM arthroplasty surgeons. This is performed as part of the annual audit and report of the SMART Registry.

\section{Data validity}

The data are extracted from the medical record and entered into a CRF (online supplemental file $3 \mathrm{a}, \mathrm{b}$ ) by trained RAs who liaise with the Registry custodian when challenges arise, and seek further detail from the surgeon who operated on the given patient whenever necessary. The CRF contains pre-established options for as many variables as possible, to minimise spelling errors and data entry errors. Prosthetic joint infections are identified and entered on the database using validated criteria. ${ }^{50}$

Th registry coordinator, who has extensive training and experience, enters data from the completed CRF onto the database. The database contains pre-established options, with minimal 'free-text' fields. The registry coordinator's office is colocated with the registry team and when potentially erroneous data entry items are identified a request for a data check with a second member of the team is initiated.

A registry custodian and principal investigator conduct routine annual audit during which the data are extensively checked and cleaned.

When researchers initiate a research project using registry data, either a custodian or data scientist extract, deidentify and clean the data. Data validity is further checked by a registry biostatistician to assess missingness. Any missingness triggers a process whereby a registry assistant is assigned the task of retrieving missing variables from the patient medical records or from the Hospitals Health Information Service. Finally, if any variable is genuinely missing (ie, data which could not be recovered from the original data collection form), researchers must apply the most appropriate method to address missingness when conducting data analyses. The method of choice for this will depend on the analysis being carried out. The biostatisticians associated with the registry provides advice on the handling of missing data for studies utilising SMART data.

Before annual linkage with AIHW, data checks are run on each variable with the aim to identify both missing and implausible data. These entries are then flagged with
Table 1 Type and number of arthroplasty procedures recorded in SMART

\begin{tabular}{lcl}
\hline Procedure type & $\begin{array}{l}\text { Knee procedures } \\
(\mathrm{n}=6812)\end{array}$ & $\begin{array}{l}\text { Hip procedures } \\
(\mathrm{n}=6343)\end{array}$ \\
\hline Primary Total & 6048 & 5811 \\
Unicompartmental & 413 & NA (knee-only) \\
Patellofemoral & 19 & N/A (knee-only) \\
Resurfacing & N/A (hip-only) & 20 \\
Revision & 332 & 512 \\
\hline
\end{tabular}

N/A, not available; SMART, St Vincent's Melbourne Arthroplasty Outcome.

the registry custodian who engages the research team to investigate.

\section{Characteristics of study participants and information on exposures and potential confounders}

Table 1 outlines a breakdown of procedure types and numbers recorded in the registry as of 30 June 2018.

Online supplemental file 4 depicts baseline demographic and comorbidity characteristics of registry patients who have undergone primary elective total knee $(\mathrm{n}=6048)$ or total hip $(\mathrm{n}=5811)$ arthroplasty between 1 January 1998 and 30 June 2018. This is presented alongside the AOANJRR and the Australian Bureau of Statistics National Health Survey (ABS NHS) characteristics wherever comparable information is available. Comparison with these national data sources indicates that registry patients are broadly representative of the Australian TJA and OA populations in terms of sociodemographic characteristics (online supplemental file 4), although with a higher proportion of females in the TKA cohort than the Australian population. Of note, a higher proportion of Registry patients live in the major cities than the ABS NHS patients. This is to be expected considering the registry is based in a large urban centre, whereas the ABS NHS sample comprises patients from all of Australia. This could contribute to the other notable difference, namely that Registry patients have a higher average socioeconomic status (SES), given that that SES decreases with increasing rurality. ${ }^{51}$ It is also important to note that the SMART Registry contains a higher proportion of ASA class 3 patients.

In Australia, public hospitals such as SVHM are funded by the government, predominantly at the state level but with federal contribution also; public patients do not incur out-of-pocket costs for surgery. Private hospitals receive federal government funding, with hospital costs supplemented by the patient's private insurance, with patients liable for any gap funding. ${ }^{52}$ Most of the data $(83 \%)$ in the registry is obtained from patients treated at the public campus of SVHM hospital. The remaining $17 \%$ is obtained from patients treated at the private SVHM campus. 


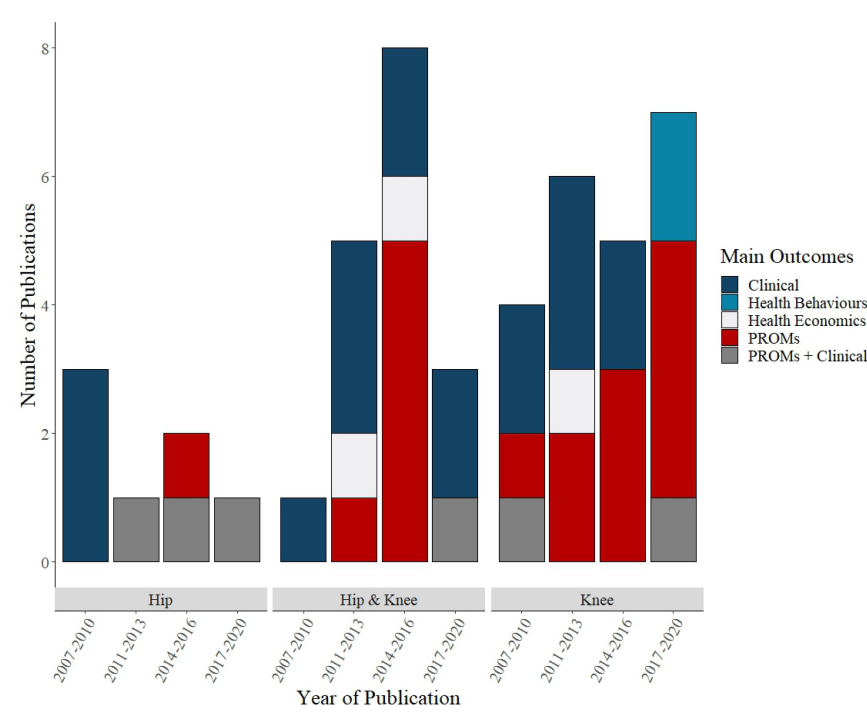

Figure 3 Registry publications over time. 'Clinical' includes complications, range of motion, transfusion, prosthetic joint infection, radiographic outcomes, PROMs, including pain and function, quality of life. PROM, patient-reported outcome measure.

\section{PATIENT AND PUBLIC INVOLVEMENT}

No patients were involved in the planning or preparation of this manuscript. Future work with SMART Registry data will take place in consultation with members of the NHMRC Centre for Research Excellence in Total Joint Replacement's Arthritis Consumer and Community Involvement Programme.

\section{FINDINGS TO DATE}

The SMART cohort has been used to investigate a range of research questions in accordance with the aims of the Registry. Registry data enable the use of a broad range of methodologies in evaluating patient characteristics, clinical outcomes and complications, patient-reported outcomes, health behaviours and healthcare costs. Registry data have been used as the sole source of information or as part of international comparative studies, and more recently has also informed multimethods studies involving qualitative evaluations. Figure 3 depicts the growth of registry publications over time. A full list of 46 publications using registry data is also provided (see online supplemental file 5).

\section{Clinical}

Dowsey et al carried out survival analysis on registry arthroplasty patients and compared with the results obtained from the same analysis using a large US-based joint replacement registry. ${ }^{53}$ Beyond 1 year, in the registry data, patients in the overweight, obese class I, II and III were each found to have lower risk of mortality as compared with normal weight patients. These findings were also obtained in the Kaiser Permanente Total Joint Replacement Registry, with the additional finding that patients were found to be at higher risk of mortality compared with normal weight patients, beyond 1 year. Despite many studies suggesting an increased risk of medical complications in obese patients undergoing TJA, even severe obesity is not independently associated with increased risk of mortality. Withholding surgery on the basis of obesity alone may not be justified in light of these findings.

\section{Health behaviours}

Bunzli et aladopted inductive thematic to identify concepts related to patient expectations of TKA. ${ }^{54}$ A preliminary framework of therapeutic misconception captured discrepancies between anticipated and actual experiences reported by a sample of patients 1-year post-TKA. By assessing and addressing patients' expectations for the TKR journey, including perioperative, postoperative and long-term outcomes, surgeons can help ensure that patients consent for surgery fully informed about what surgery involves and what their likely outcomes will be.

\section{Health costs}

Peel et al developed a negative binomial regression model to quantify factors associated with costs in the first 30 days following TJA. ${ }^{3}$ The findings were extrapolated to the Australian population and it was found that preventable postoperative complications were major cost drivers, with surgical site infection (SSI) adding \$A97 million, venous thromboembolism adding \$A66million and delirium adding \$A64million to the total annual cost of TJA in Australia, which already exceeds \$A1 billion. Reducing the rate of postoperative complications in TJA represents an opportunity to make a substantial impact on the overall cost burden on the healthcare system.

\section{PROMs}

Dowsey et al used Latent Class Growth Analysis to classify groups of patients according to their trajectory of knee pain/ function over $1-5$ years post-TKA. ${ }^{55}$ Modifiable predictors of poor response; namely poor function and/or moderate-tosevere pain, were identified: baseline comorbidity, physical and mental well-being and obesity. Clinicians can use these findings to identify patients with a higher likelihood of poor surgical response, and incorporate this information into a shared decision-making process.

\section{FUTURE PLANS}

The 2019 MBS and PBS person-level data linkage will facilitate further investigation into areas including prescribing patterns in arthroplasty patients, as well as chronic condition management and health service utilisation. See online supplemental file 6 for the full list of MBS and PBS items included in linkage. Linked MBS items provide an all-round understanding of the timing and nature of healthcare utilisation of arthroplasty patients including rehabilitation, rehospitalisation and emergency department presentations, before and after surgery. PBS Anatomical Therapeutic Chemical codes allow the calculation of measures such as the Rx-Risk-V for potential arthroplasty outcomes and complications. The 
Rx-Risk-V is a prescription-based comorbidity index that uses patients' medical history, and inpatient and outpatient pharmacy data to identify the presence of 45 conditions. ${ }^{56}$ It is valid measure for predicting future mortality, healthcare utilisation and healthcare costs. ${ }^{57}$ With approval obtained for four future annual linkages, we aim to incorporate this linked administrative data as an enduring resource in the registry.

Further additions and linkages are also planned. Annual linkage of hospital cost data is being implemented to better understand healthcare costs related to OA and arthroplasty surgery, essential for establishing cost effectiveness. The Clavien-Dindo classification system for standardised reporting of a broad range of surgical complications is currently being added to the Registry. ${ }^{58}$ As well as being added to the ongoing data collection process, this system will also be applied retrospectively to existing Registry records. This will improve the auditing process for the registry and improve the quality of publications resulting from research involving registry data, by providing more detailed information on complications and enabling comparisons across surgical specialties.

Concerning the long-term life of the registry, it is linked with permanent clinical activity in perpetuity. As an active research-focused institution, orthopaedic clinicianresearchers engaged in research and clinical practice at SVHM work closely with the registry custodians. The staff governance structure also ensures appropriate succession planning that will take effect at the appropriate time.

Regarding future plans for the logistical and pragmatic aspects of data collection at SVHM, the SMART Registry research team has no direct input into decisions made by hospital administration, however, as a group actively involved in patient-centred research, it is hoped Registry researchers will be useful in informing decisions pertaining to data collection practices at the hospital. This, in turn, may alter the way in which research is conducted.

\section{STRENGTHS AND LIMITATIONS}

All elective hip and knee arthroplasty patients at the hospital are included, and a range of PROMs are recorded with longterm follow-up and high proportion of patients participating in follow-up. This facilitates robust longitudinal analysis of outcomes at the individual patient level while also generating a substantial cohort of patients whose long-term outcomes can be compared. Data collection is also ongoing, with extensive training and mentoring in place for staff working on the registry. There is a monthly meeting at which registry staff, including the custodian, convene to discuss workflow and any issues arising such as difficulty obtaining follow-up questionnaire responses. Where data collection or quality assurance issues arise, research staff can contact the orthopaedic clinic and hospital staff, and access both physical and electronic copies of original patient medical records. For example, this was done to update the comorbidity data such that the CCI could be accurately calculated. Beyond these frequent routine, and as-needed, data quality assurance practices, the registry custodian also conducts a formal annual audit in order to identify any concerns that may have arisen over the course of the year. The growth of the registry, in both size and comprehensiveness, has reached the point where linkage with national federally subsidised healthcare and prescribed medication records databases (ie, MBS and PBS) are possible. This opens avenues of future research that will better inform the evidence base for policy and practice changes to improve patient care.

In light of the many strengths of the registry, it is important to note that it is institutional and not national. The registry cohort is likely generalisable to the Australian TJA population (see online supplemental file 4), however, differences such as the higher proportion of females and ASA class 3 patients in the Registry TKA cohort compared with the AOANJRR cohort must be noted. Furthermore, this is an institutional registry from a tertiary referral centre in Melbourne, Australia, and the findings may not be generalisable to other settings. However, collaborative projects with the Kaiser Permanente group in the USA, as well as the Swedish Arthroplasty Registry, demonstrated an exceptionally high degree of congruence between these databases and the SMART Registry. ${ }^{5359}$ While comparability with other databases needs to be assessed on a case-by-case basis relying on the broad range of variables captured (see online supplemental file 1), these findings indicate that findings from research with SMART Registry data are applicable at least to some separate settings. Also, since the Registry is not linked to state-based databases such as the Victorian Emergency Minimum Dataset and the Victorian Admitted Episodes Dataset, it is not possible to determine with absolute certainty whether Registry patients were subsequently admitted to other hospitals, or experienced an emergency department visit at another hospital. ${ }^{60}{ }^{61}$ Despite this limitation, routine follow-up appointments commencing at 6 weeks postsurgery are mandatory for St Vincent's Hospital Melbourne arthroplasty patients, and at this appointment the consultant surgeon who performed the surgery interviews the patient regarding the details of their postoperative course including emergency department visits and admissions to hospitals other than St Vincent's. Further routine follow-up occurs at 3 or 6 months, at 1 year, 2 or 3 years, 5 years and then 10 years; the individual patient's clinical progression is taken into account when arranging follow-up.

\section{COLLABORATION}

All registry data are stored securely, and confidentiality is upheld. The data are accessible only by members of the research team who have been approved by the Human Research Ethics Committees of St. Vincent's Hospital Melbourne (HREC-A 100/14). The investigators do not receive external funding to maintain the registry, and as such lack the capacity and infrastructure for open data sharing. Researchers interested in collaboration are welcome to contact MMD (mmdowsey@unimelb.edu.au), principal investigator. All requests are considered subject to funding support and ethics approval.

Author affiliations

${ }^{1}$ Department of Surgery, The University of Melbourne St Vincent's, Fitzroy, Victoria, Australia 
${ }^{2}$ Department of General Practice, The University of Melbourne, Carlton, Victoria, Australia

${ }^{3}$ Centre for Health Policy, Melbourne School of Population and Global Health, The University of Melbourne, Melbourne, Victoria, Australia

${ }^{4}$ Melbourne School of Population and Global Health, The University of Melbourne Centre for Health Equity, Melbourne, Victoria, Australia

${ }^{5}$ Centre for Adolescent Health, Murdoch Childrens Research Institute, Parkville, Victoria, Australia

${ }^{6}$ Department of Orthopaedics, St Vincent's Hospital Melbourne Pty Ltd, Fitzroy, Victoria, Australia

Correction notice This article has been corrected since it first published. The provenance and peer review statement has been included.

Acknowledgements We acknowledge the researchers and staff members who contribute to, and maintain, the SMART Registry.

\section{Collaborators N/A}

Contributors PFC and MMD wrote the original research protocol, sought ethics approval, designed the Registry and oversaw data acquisition. PFC, MMD, DG, JY, JK, ST, CSC and CSh have made substantial contributions to the analysis and interpretation of data. DG drafted the manuscript, with PFC, MMD, JY, JK, ST, CSc and CSh providing intellectual content. PFC, MMD, DG, JY, JK, ST, CSC and CSh have reviewed and approved the final manuscript, and all agree to be accountable for all aspects of the manuscript and will work together to ensure questions relating to the accuracy and integrity of any part of it are appropriately investigated and resolved.

Funding The authors have not declared a specific grant for this research from any funding agency in the public, commercial or not-for-profit sectors.

Competing interests None declared.

Patient consent for publication Not required.

Ethics approval The SMART Registry has been approved by the Human Research Ethics Committees of St. Vincent's Hospital Melbourne (HREC-A 100/14) and is registered with the Health Sciences Human Ethics Sub-Committee, Melbourne School of Population and Global Health, University of Melbourne. Following peer review, the linkage with PBS and MBS data was approved by the AlHW Ethics Committee (E02018/2/450)

Provenance and peer review Not commissioned; externally peer reviewed.

Data availability statement Data are available on reasonable request. All Registry data, comprising identifiable patient-level information, are stored securely, and confidentiality is upheld. The data are accessible only by members of the research team who have been approved by the Human Research Ethics Committees of St. Vincent's Hospital Melbourne (HREC-A 100/14). The investigators do not receive external funding to maintain the registry, which limits the capacity for open data sharing. Requests for SMART data should be made to the corresponding author and will be considered by the Registry Custodians. Consent for data sharing has not been obtained and therefore ethics approval would be required from the study institution for future use of SMART data.

Supplemental material This content has been supplied by the author(s). It has not been vetted by BMJ Publishing Group Limited (BMJ) and may not have been peer-reviewed. Any opinions or recommendations discussed are solely those of the author(s) and are not endorsed by BMJ. BMJ disclaims all liability and responsibility arising from any reliance placed on the content. Where the content includes any translated material, BMJ does not warrant the accuracy and reliability of the translations (including but not limited to local regulations, clinical guidelines, terminology, drug names and drug dosages), and is not responsible for any error and/or omissions arising from translation and adaptation or otherwise.

Open access This is an open access article distributed in accordance with the Creative Commons Attribution Non Commercial (CC BY-NC 4.0) license, which permits others to distribute, remix, adapt, build upon this work non-commercially, and license their derivative works on different terms, provided the original work is properly cited, appropriate credit is given, any changes made indicated, and the use is non-commercial. See: http://creativecommons.org/licenses/by-nc/4.0/.

\section{ORCID iDs}

Daniel Gould http://orcid.org/0000-0002-0423-5822

Sharmala Thuraisingam http://orcid.org/0000-0002-9450-1302

Cade Shadbolt http://orcid.org/0000-0002-0937-2412

Josh Knight http://orcid.org/0000-0001-5268-8263

Jesse Young http://orcid.org/0000-0001-5702-372X
Chris Schilling http://orcid.org/0000-0002-1747-7249

Michelle M Dowsey http://orcid.org/0000-0002-9708-5308

\section{REFERENCES}

1 Ethgen $\mathrm{O}$, Bruyère $\mathrm{O}$, Richy $\mathrm{F}$, et al. Health-related quality of life in total hip and total knee arthroplasty. A qualitative and systematic review of the literature. J Bone Joint Surg Am 2004;86:963-74.

2 Neuprez A, Neuprez AH, Kaux JF. Total joint replacement improves pain, functional quality of life, and health utilities in patients with latestage knee and hip osteoarthritis for up to 5 years. Clin Rheumatol 2019.

3 Peel TN, Cheng AC, Liew D, et al. Direct hospital cost determinants following hip and knee arthroplasty. Arthritis Care Res 2015;67:782-90.

4 Bozic KJ, Ward L, Vail TP, et al. Bundled payments in total joint arthroplasty: targeting opportunities for quality improvement and cost reduction. Clin Orthop Relat Res 2014;472:188-93.

5 Cutler DM, Ghosh K. The potential for cost savings through bundled episode payments. N Engl J Med 2012;366:1075-7.

6 Inacio MCS, Graves SE, Pratt NL, et al. Increase in total joint arthroplasty projected from 2014 to 2046 in Australia: a conservative local model with international implications. Clin Orthop Relat Res 2017;475:2130-7.

7 Kurtz S, Ong K, Lau E, et al. Projections of primary and revision hip and knee arthroplasty in the United States from 2005 to 2030. J Bone Joint Surg Am 2007;89:780-5.

8 Halawi MJ, Jongbloed W, Baron S, et al. Patient Dissatisfaction After Primary Total Joint Arthroplasty: The Patient Perspective. J Arthroplasty 2019;34:1093-6.

9 Gunaratne R, Pratt DN, Banda J, et al. Patient Dissatisfaction following total knee arthroplasty: a systematic review of the literature. $J$ Arthroplasty 2017;32:3854-60.

10 Okafor L, Chen AF. Patient satisfaction and total hip arthroplasty: a review. Arthroplasty 2019;1:6.

11 Gandhi R, Davey JR, Mahomed NN. Predicting patient Dissatisfaction following joint replacement surgery. J Rheumatol 2008;35:2415-8.

12 Wilson I, Bohm E, Lübbeke A, et al. Orthopaedic registries with patient-reported outcome measures. EFORT Open Reviews 2019;4:357-67.

13 Lübbeke A, Silman AJ, Prieto-Alhambra D, et al. The role of national registries in improving patient safety for hip and knee replacements. BMC Musculoskelet Disord 2017;18:414.

14 Statistics ABo. 3222.0 - Population Projections, Australia, 2017 (base) - 2066, 2018. Available: https://www.abs.gov.au/AUSSTATS/ abs@.nsf/Latestproducts/3222.0Main\%20Features72017\%20( base)\%20-\%202066?opendocument\&tabname=Summary\&prodno= $3222.0 \&$ issue $=2017 \% 20$ (base) $\% 20-\% 202066 \&$ num $=\& v i e w=$ [Accessed 2 Apr 2020].

15 Australian Orthopaedic Association National Joint Replacement Registry. Australian orthopaedic association national joint replacement registry, 2020. Available: https://aoanjrr.sahmri.com/ procedures-reported [Accessed 25 Aug 2020].

16 Kellgren JH, Lawrence JS. Radiological assessment of osteoarthrosis. Ann Rheum Dis 1957;16:494-502.

17 Adhikari P. Socio-Economic indexes for areas: introduction, use and future directions. Canberra: Australian Bureau of Statistics, 2006: 1-37.

18 Do H, Care A. Measuring remoteness: accessibility/remoteness index of Australia (ARIA). Revised Ed 2001.

19 Statistics ABo. ASGC remoteness classification: purpose and use. Census paper NO: 03/01, 2003.

20 WHO. Obesity: preventing and managing the global epidemic. World Health Organization, 2000.

21 Daabiss M. American Society of Anaesthesiologists physical status classification. Indian J Anaesth 2011;55:111.

22 Wolters $U$, Wolf T, Stützer $\mathrm{H}$, et al. ASA classification and perioperative variables as predictors of postoperative outcome. $\mathrm{Br} J$ Anaesth 1996;77:217-22.

23 Owens WD, Felts JA, Spitznagel EL. ASA physical status classifications: a study of consistency of ratings. Anesthesiology 1978;49:239-43.

24 Gliklich RE DN, Leavy MB, eds. Data elements for registries. Registries for evaluating patient outcomes: A user's guide. 3rd edn. Rockville: Agency for Healthcare Research and Quality, 2014

25 Surgeons RACo. A guide by the Royal Australasian College of surgeons: surgical audit and peer review, 2013.

26 Association AO. Annual report. Adelaide, Australia: South Australian Health and Medical Research Institute, 2019. 
27 Noble PC, Scuderi GR, Brekke AC, et al. Development of a new knee society scoring system. Clin Orthop Relat Res 2012;470:20-32.

28 Ramkumar PN, Harris JD, Noble PC. Patient-reported outcome measures after total knee arthroplasty. Bone Joint Res 2015;4:120-7.

29 Nilsdotter A, Bremander A. Measures of hip function and symptoms: Harris hip score (HHS), hip disability and osteoarthritis outcome score (HOOS), Oxford hip score (OHS), Lequesne index of severity for osteoarthritis of the hip (LISOH), and American Academy of orthopedic surgeons (a. Arthritis Care Res 2011;63:S200-7.

30 Gossec L, Paternotte S, Maillefert JF, et al. The role of pain and functional impairment in the decision to recommend total joint replacement in hip and knee osteoarthritis: an international crosssectional study of 1909 patients. Report of the OARSI-OMERACT Task force on total joint replacement. Osteoarthritis and Cartilage 2011;19:147-54.

31 Bellamy N, Buchanan WW, Goldsmith $\mathrm{CH}$, et al. Validation study of WOMAC: a health status instrument for measuring clinically important patient relevant outcomes to antirheumatic drug therapy in patients with osteoarthritis of the hip or knee. $J$ Rheumatol 1988;15:1833-40.

32 Bourne RB, Chesworth BM, Davis AM, et al. Patient satisfaction after total knee arthroplasty: who is satisfied and who is not? Clin Orthop Relat Res 2010;468:57-63.

33 Ko Y, Lo N-N, Yeo S-J, et al. Comparison of the responsiveness of the SF-36, the Oxford knee score, and the knee society clinical rating system in patients undergoing total knee replacement. Qual Life Res 2013;22:2455-9.

34 Bachmeier CJM, March LM, Cross MJ, et al. A comparison of outcomes in osteoarthritis patients undergoing total hip and knee replacement surgery. Osteoarthritis and Cartilage 2001;9:137-46.

35 Quintana JM, Escobar A, Bilbao A, et al. Responsiveness and clinically important differences for the WOMAC and SF-36 after hip joint replacement. Osteoarthritis and Cartilage 2005;13:1076-83.

36 McConnell S, Kolopack P, Davis AM. The Western Ontario and McMaster universities osteoarthritis index (WOMAC): a review of its utility and measurement properties. Arthritis \& Rheumatism 2001;45:453-61.

37 Ware J, Kosinski M, Keller SD. A 12-Item short-form health survey: construction of scales and preliminary tests of reliability and validity. Med Care 1996;34:220-33.

38 Kazis LE, Miller DR, Skinner KM, et al. Applications of methodologies of the Veterans health study in the Va healthcare system: conclusions and summary. J Ambul Care Manage 2006;29:182-8.

39 Brazier JE, Roberts J. The estimation of a preference-based measure of health from the SF-12. Med Care 2004;42:851-9.

40 Hall WH, Ramachandran R, Narayan S, et al. An electronic application for rapidly calculating Charlson comorbidity score. BMC Cancer 2004;4:94.

41 Bjorgul K, Novicoff WM, Saleh KJ. Evaluating comorbidities in total hip and knee arthroplasty: available instruments. J Orthop Traumatol 2010;11:203-9.

42 ANAO. Integrity of medicare enrolment data. Canberra ACT: Australian National Audit Office, 2005.

43 Health AGDo. MBS online - medicare benefits schedule, 2020. Available: http://www.mbsonline.gov.au/internet/mbsonline/ publishing.nsf/Content/Home [Accessed 17 Mar 2020].
44 Commissioner AGOotAl. Publication of MBS/PBS data Commissioner inititated investigation report, 2018. Available: https:// www.oaic.gov.au/privacy/privacy-decisions/investigation-reports/ mbspbs-data-publication/ [Accessed 20 Apr 2020].

45 Health AGDo. The Pharmacerutical benefits scheme, 2020. Available: http://www.pbs.gov.au/pbs/home;jsessionid=i129gwsetg703u9c vmbjxhv6 [Accessed 17 Mar 2020].

46 Mellish L, Karanges EA, Litchfield MJ, et al. The Australian pharmaceutical benefits scheme data collection: a practical guide for researchers. BMC Res Notes 2015;8:634.

47 Welfare AloHa. Pharmaceutical benefits scheme (PBS) data collection, 2019. Available: https://www.aihw.gov.au/about-our-data/ our-data-collections/pharmaceutical-benefits-scheme [Accessed 16 Apr 2019].

48 Lizzio VA, Dekhne MS, Makhni EC. Electronic patient-reported outcome collection systems in orthopaedic clinical practice. JBJS Rev 2019;7:e2.

49 de Steiger RN, Graves SE. Orthopaedic registries: the Australian experience. EFORT Open Rev 2019;4:409-15.

50 Parvizi J, Tan TL, Goswami K, et al. The 2018 definition of periprosthetic hip and knee infection: an evidence-based and validated criteria. $J$ Arthroplasty 2018;33:1309-14.

51 Welfare AloHa. Rural, regional and remote health: indicators of health. Canberra: AlHW, 2005

52 Peel TN, Dowsey MM, Buising KL, et al. Cost analysis of debridement and retention for management of prosthetic joint infection. Clin Microbiol Infect 2013;19:181-6.

53 Dowsey MM, Choong PFM, Paxton EW, et al. Body mass index is associated with all-cause mortality after THA and TKA. Clin Orthop Relat Res 2018;476:1139-48.

54 Bunzli S, O'Brien P, Klem N, et al. Misconceived expectations: patient reflections on the total knee replacement journey. Musculoskeletal Care 2020;18:415-24.

55 Dowsey MM, Smith AJ, Choong PFM. Latent class growth analysis predicts long term pain and function trajectories in total knee arthroplasty: a study of 689 patients. Osteoarthritis Cartilage 2015;23:2141-9.

56 Sloan KL, Sales AE, Liu C-F, et al. Construction and characteristics of the RxRisk-V: a VA-adapted pharmacy-based case-mix instrument. Med Care 2003:41:761-74.

57 Pratt NL, Kerr M, Barratt JD, et al. The validity of the Rx-Risk comorbidity index using medicines mapped to the anatomical therapeutic chemical (ATC) classification system. BMJ Open 2018;8:e021122.

58 Clavien PA, Barkun J, de Oliveira ML, et al. The clavien-dindo classification of surgical complications: five-year experience. Ann Surg 2009;250:187-96.

59 Dowsey MM, Robertsson O, Sundberg M, et al. Variations in pain and function before and after total knee arthroplasty: a comparison between Swedish and Australian cohorts. Osteoarthritis Cartilage 2017;25:885-91.

60 Services VSGDoHaH. Victorian emergency minimum dataset June 2019, 2019. Available: https://www2.health.vic.gov.au/hospitals-andhealth-services/data-reporting/health-data-standards-systems/datacollections/vemd [Accessed $20 \mathrm{Apr} 2020]$.

61 Services VSGDoHaH. Victorian admitted episodes dataset. July 2019, 2019. Available: https://www2.health.vic.gov.au/hospitals-andhealth-services/data-reporting/health-data-standards-systems/datacollections/vaed [Accessed 20 Apr 2020]. 